\title{
Development and Feasibility of a Home Pulmonary Rehabilitation Program With Health Coaching
}

\author{
Roberto P Benzo MD MSc, Kevin M Kramer PhD, Johanna P Hoult MA CCRP, \\ Paige M Anderson, Ivonne M Begue, and Sara J Seifert MPH
}

\begin{abstract}
BACKGROUND: Pulmonary rehabilitation (PR) is an effective intervention for COPD. However, traditional center-based PR programs suffer from low uptake. Home-based PR is a viable solution, but few studies have shown the effectiveness of remote PR, as there is a scarcity of systems that can be easily adopted in clinical practice. The aim of this report is to communicate the development and feasibility of a home PR program that includes commercially available technology that allows the PR health coach to follow the patient through his or her PR process and to present the design of a prospective clinical trial. METHODS: We developed a home PR system that includes a computer tablet, an activity monitor, and an oximeter connected to a cloud server. The home PR consists of 12 min of walking and 6 full-body exercises, to be completed $6 \mathrm{~d} /$ week, plus weekly telephone calls with the PR health coach. Two pilot studies were conducted in subjects with moderate-to-severe COPD. The first aimed to fine-tune the system development $(N=3)$, and the second tested the program feasibility of the 8 -week program $(N=12)$. RESULTS: In pilot study 1 , PR monitoring data from the subjects' home PR sessions were transmitted to the health coach application successfully. On a 10-point scale, participants rated the system as helpful (median $=8$, interquartile range 8-9) and simple to use (median $=10$, interquartile range 9-10). In pilot study 2, adherence \pm SD for prescribed use was $87 \pm 0.24 \%$. Overall, participants gave the home PR system a rating of $6.2 \pm 0.94$ on a 7-point scale. CONCLUSIONS: A home PR program was developed that integrated health coaching and a home PR system that facilitated remote monitoring. Pilot testing indicated that the program is well-developed and feasible in a population of individuals with COPD. (ClinicalTrials.gov registration NCT02999685.) Key words: COPD; pulmonary rehabilitation; health coaching; quality of life; motivational interviewing; self-management; home-based pulmonary rehabilitation. [Respir Care 2018;63(2):131-140. @ 2018 Daedalus Enterprises]
\end{abstract}

\section{Introduction}

Pulmonary rehabilitation (PR) is an effective intervention for COPD. ${ }^{1}$ However, uptake of PR is low due to patient frailty, transportation issues, and access. ${ }^{2}$ Home-

\footnotetext{
Dr Benzo, Ms Hoult, and Ms Anderson are affiliated with the Mindful Breathing Laboratory, Division of Pulmonary and Critical Care Medicine, and Ms Begue is affiliated with Community-Based Research, Mayo Clinic, Rochester, Minnesota. Dr Kramer and Ms Seifert are affiliated with the Minnesota Health Solutions Corporation, Saint Paul, Minnesota.

Dr Benzo, Ms Hoult, Ms Seifert, and Dr Kramer are supported by National Institutes of Health Grant SBIRHL 114162-2 (to Ms Seifert [PI] and Dr Benzo [clinical trial PI]). Ms Seifert has disclosed a relationship with NovuHealth. The other authors have disclosed no conflicts of interest.
}

based rehabilitation has been introduced in recent years to palliate the lack of feasibility for many patients to attend traditional center-based PR programs. ${ }^{3,4}$ However, concerns remain about how to monitor patients completing PR at home for both safety and adherence. There is growing access to remote monitoring of people

\footnotetext{
Supplementary material related to this paper is available at http:// www.rcjournal.com.

Correspondence: Roberto Benzo MD MSc, Mindful Breathing Laboratory, Division of Pulmonary and Critical Care, Mayo Clinic, 200 First Street SW, Gonda 18-440, Rochester, MN 55902. E-mail: benzo.roberto@mayo.edu.
}

DOI: $10.4187 /$ respcare. 05690 


\section{Home-Based Pulmonary Rehab and Health Coaching for COPD}

at home, or anywhere, which may promote an effective integration of rehabilitation routines into the daily life of the patient while allowing monitoring. In addition to

See the Related Editorial on Page 253

the benefits for patients of completing PR, the field is currently aiming for heavier modification effects over the long term. Only a few studies have shown the effectiveness of remote PR, ${ }^{1,3,4}$ which may be due to the scarcity of systems that can be easily adopted in clinical practice.

\section{Aiming for Home Pulmonary Rehabilitation Programs With Lasting Results}

Long-term adherence with activities learned in PR has been problematic for conventional PR, as the effects of PR have historically diminished over time, with deterioration in both exercise capacity and health-related quality of life within 6-12 months of program completion. ${ }^{5}$ One key reason for this decline is the lack of behavior change as a result of PR. It is now postulated that undertaking PR in the home may promote effective behavior change through integration of physical activity routines into daily life. ${ }^{6}$ Self-management support, which creates ideal conditions for behavior change, is now recommended by PR guidelines as an essential part of chronic care, particularly in PR programs. ${ }^{1,7} \mathrm{~A}$ large randomized study of COPD self-management support identified lack of patient engagement as the core problem in adopting new behaviors. ${ }^{8}$ In that study, significant effects on health-related quality of life and health-care utilization after the self-management support intervention occurred only in participants who were actively engaged in their care. Brief advice is not enough to promote behavior change; meaningful engagement is necessary. ${ }^{9}$

\section{Health Coaching: A Potential Synergy With Pulmonary Rehabilitation for Promoting Behavior Change}

In our previous work, we developed and tested an effective program for self-management support in COPD through health coaching ${ }^{10}$ rooted in motivational interviewing ${ }^{10,11}$ that decreased COPD rehospitalizations ${ }^{12}$ and produced a sustained improvement in quality of life at 6 and 12 months. Motivational interviewing is an evidencebased, efficient method of guiding individuals into sustained behavior change. ${ }^{13}$ Feedback is critical: Motivational interviewing-based health coaching, proven effective in COPD, ${ }^{12}$ can translate to higher self-efficacy, subsequently promoting better self-management support and improved quality of life. ${ }^{14,15}$

\section{QUICK LOOK}

\section{Current knowledge}

Home-based pulmonary rehabilitation (PR) may be equally effective in improving fitness and quality of life as a traditional center-based program for COPD patients and may represent a valid alternative to centerbased PR that currently lacks enough adoption due to multiple factors. Despite the potential benefit of home $\mathrm{PR}$, there is a scarcity of home-based PR systems that can be easily adopted in clinical practice. Having effective and simple systems for home PR readily available will facilitate the implementation of home-based $\mathrm{PR}$ in clinical practice.

\section{What this paper contributes to our knowledge}

A home PR program was developed that integrated health coaching and a home PR system that facilitated remote monitoring. The home PR program was found to be feasible and well-accepted by targeted users. The system used off-the-shelf technology that will facilitate adoption, and most important early results reported indicated a high adherence to the prescribed PR. The latter may fill a practice gap in PR by increasing adherence and completion of PR programs.

\section{The Ideal Home-Based Pulmonary Rehabilitation System}

The ideal system for a home PR program requires easyto-use and accurate technology to monitor physiological parameters, the promotion of patient engagement, and a curriculum of simple exercises to be performed safely at home.

The aim of this report is to communicate the development and feasibility of a home PR system and to provide preliminary results from the initial pilot studies that combined the system with a behavioral program. Finally, we present the design of a prospective randomized trial funded by the National Institutes of Health that will test the efficacy of the entire home-based program (system plus health coaching).

\section{Methods}

\section{The Home-Based Pulmonary Rehabilitation Program}

The proposed home PR program (Fig. 1) is composed of, first, the system that consists of off-the-shelf components, including a commercially available tablet computer, an activity monitor, and a pulse oximeter, all accompanied 


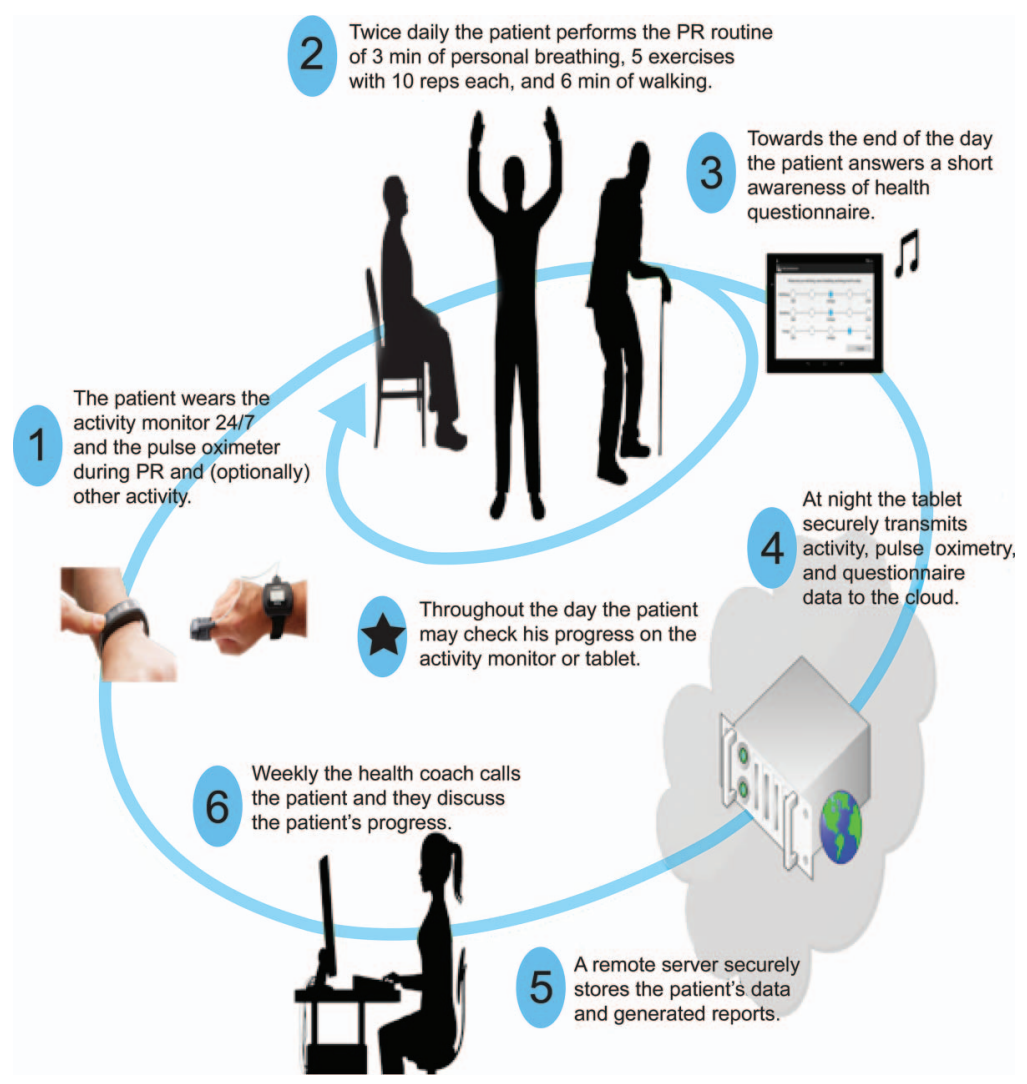

Fig. 1. The proposed pulmonary rehabilitation (PR) program, including the system and health coaching.

by custom software, and, second, the PR health coaching. Participants are to complete PR exercises by following along with videos provided on the tablet. While completing the exercises, participants wear the pulse oximeter to provide physiological feedback. After completing their exercises, participants complete a 3-item wellness questionnaire, which asks the participants to rate their well-being, ${ }^{13}$ ease of breathing, and energy level. Participants wear the activity monitor continuously to provide daily step counts and motivation.

The activity monitor and pulse oximeter wirelessly communicate with the tablet via Bluetooth, allowing the participants to track their progress. Each night, the tablets securely transmit the device data and well-being questionnaire answers via a cellular network to a remote secure cloud, which securely stores the data and generates reports for the PR health coach to follow the patient through the process and assess adherence. Each week, PR health coaches call the participants to discuss their progress.

Pulmonary Rehabilitation Exercises. Two authors (RPB and IMB) developed an 8-week program that includes an exercise routine comprised of 6 full-body, lowintensity exercises, with the option of selecting seated or standing exercises. Additionally, participants are asked to complete at least $12 \mathrm{~min}$ of focused, slow walking. Par- ticipants will be asked to complete the focused walking and exercise routine at least $6 \mathrm{~d} /$ week.

There are videos demonstrating each exercise, as well as the focused walking, and participants are encouraged to exercise and walk at the pace of the videos provided. Each subject is asked to wear a fingertip pulse oximeter while completing the prescribed exercise routine, to provide $\mathrm{S}_{\mathrm{pO}_{2}}$ and heart rate feedback as well as to monitor adherence.

Activity Monitor. The Vívofit 2 activity monitor (Garmin, Schaffhausen, Switzerland) was selected as suitable for this population to provide daily monitoring and motivation in an easy-to-use system. There is a clip-on version for those who use a walker. The activity monitor chiefly provides a summary of daily steps, a key physical activity outcome in COPD. ${ }^{14-17}$

The Vívofit 2 was integrated into the system after applying for test credentials, demonstrating competency on Garmin's test environment by developing an Android test application, applying for production credentials, and porting the test application's functionality into the system.

The patient interface on the computer tablet passively retrieves activity data from Garmin's cloud, processes the raw activity data into an efficient form, stores the processed data in a local database, and uploads the processed 


\section{Home-Based Pulmonary Rehab and Health Coaching for COPD}

data to a secure external cloud for participant activity reports and for the more detailed PR health coach activity reports. Participants are able to view their daily steps (see the supplementary materials at http://www.rcjournal.com) from the activity monitor and are not required to complete any special actions for the data to upload.

Pulse Oximeter. The WristOx ${ }_{2}$ model 3150 (Nonin Medical, Plymouth, Minnesota) was selected because it securely straps to the wrist and does not lower perfusion, it tolerates motion artifacts, and it wirelessly streams pulse oximetry data. The WristOx ${ }_{2}$ supports 2 Bluetooth profiles. We selected to use the serial port profile due to its simplicity and efficiency. Next, we developed a standalone test application that performs the following functions: (1) connects to the WristOx ${ }_{2}$; (2) configures the WristOx ${ }_{2}$; (3) synchronizes with the WristOx ${ }_{2}$ data stream; (4) parses the WristOx $x_{2}$ data stream; (5) displays the participant's heart rate, $\mathrm{S}_{\mathrm{pO}_{2}}$, and device flags; (6) logs the pulse oximetry data for off-line analysis; and (7) disconnects from the WristOx ${ }_{2}$ when the participant removes his or her finger or taps a disconnect button.

For configuration, the application sets the $\mathrm{WristOx}_{2}$ date and time, specifies which data format the WristOx $x_{2}$ should stream, and instructs the WristOx $\mathrm{x}_{2}$ to turn on and off when the participant inserts and removes his or her finger into/from the sensor, respectively. The researchers selected format 8 , which streams the participant's heart rate, $\mathrm{S}_{\mathrm{pO}_{2}}$, and device flags once per second.

Although the monitoring of the patient will not be in real time, any significant physiologic abnormality measured during rehabilitation and transmitted to the server $\left(\mathrm{S}_{\mathrm{pO}_{2}}\right.$ saturation $<85 \%$ or heart rate $>140$ or $<40$ beats $\left./ \mathrm{min}\right)$ will prompt a message to the coach to initiate a call to the participant to stop the process and investigate the event. The principal investigator, RPB, will also be alerted anytime such a physiologic abnormality is measured and ensure that the appropriate response is initiated.

Tablet Computer and Participant Software Application. The Ellipsis 10 tablets (Verizon, New York) were selected to host the patient interface as well as function as a telemedicine hub for the activity monitor and pulse oximeter. This tablet has a 10-inch display whose resolution is $1,920 \times 1,200$ pixels. These tablets have $3 \mathrm{G} / 4 \mathrm{G}$ LTE cellular radios so that participants are not required to have broadband Internet or add the tablet to their home network.

The custom patient interface has a default home screen (see the supplementary materials) that includes a daily to-do list and messages from the PR health coach. Most of the messages are preprogrammed system messages, such as a reminder to return the study device in its original mailing package. To initiate one of the activities from the to-do list, the user selects the desired activity by tapping the screen. For instance, selecting Complete Daily Exercise (see the supplementary materials) from the To-Do column initiates a video guiding the user through the PR exercises. Before displaying the exercise and walking videos, which are asked to be followed, the application verifies that the participant is wearing the pulse oximeter to ensure physiologic measures and adherence, as evidenced by the expected increase in heart rate during exercise. When the participant finishes the selected activity, a check mark appears to the left of the item to indicate that it has been completed.

Participants can select from 4 additional tabs, located at the top of the screen: Exercises \& Walking, Check In, My Journey, and Help. Under the Check In tab (see the supplementary materials), participants can access the 3 -item questionnaire on their well-being that they are asked to complete each day of the $6 \mathrm{~d} /$ week that they are asked to perform the exercises. When the option My Journey is selected, it provides a custom report that includes trend and detailed data on the subject's daily activity and physiological measures, drawn from the activity monitor and pulse oximeter. The final option on the upper bar is the Help option (see the supplementary materials), which includes how-to videos for the equipment as well as an opportunity to submit questions or comments to the study coordinator about any problems they may be experiencing.

The patient interface is an Android software application that securely stores and transfers de-identified activity, pulse oximetry, and questionnaire data to the cloud. Additionally, this service fetches and transmits the actual weather data to the cloud for the PR health coach to view. The tablet interface was designed for older adults with the intent that it be easy to use and limit extraneous information or choices. Participants can access only the PR program on the tablet and do not have the ability to access other programs or the Internet.

Health Coaching. Each subject receives a weekly coaching call to discuss rehabilitation and health progress with a trained PR health coach. The calls will be structured using motivational interviewing and will follow the published protocol. ${ }^{10,12}$ The coach will review the data from the activity-monitoring system with the subject specifically daily steps, exercise duration and frequency, time of day of the rehabilitation exercise, and physiologic measures (oxygen saturation and heart rate). The coach will also review the answers to the daily well-being questionnaire. Collaboratively, goals for rehabilitation exercise for the following week will be set, based on the information gathered and participants' personal preferences.

Each call lasts about $12 \mathrm{~min}$. All calls are recorded to ensure adherence with the protocol. To complete the in- 


\section{Home-Based Pulmonary Rehab and Health Coaching for COPD}

tervention, each subject needs to complete telephone calls with a PR health coach in at least 6 of the 8 weeks. At the end of the 8-week intervention period, a qualitative semistructured interview will be conducted with each subject by the PR health coach to gather information on the strengths and weaknesses of the process and intervention. Details of the training of the PR health coaches have been published previously. ${ }^{10}$

Health Coach Software Application. A custom PC software application was created for the PR health coaches to monitor patient progress. This custom program includes an opening screen (see the supplementary materials) that lists all participants and their respective summary data about the activity monitor, exercises and walking, and the check-in questionnaire. This screen is color-coded and will indicate flags (eg, participant did not complete exercises for a certain number of days) or alerts $\left(\mathrm{S}_{\mathrm{pO}_{2}}\right.$ or heart rate outside of predefined bounds) to make it easy for PR health coaches to identify subject concerns. Each participant can be selected to see a weekly report (see the supplementary materials) on daily weather (as better weather promotes more physical activity), steps, duration of exercise and walking, pulse oximeter data (heart rate and $\mathrm{S}_{\mathrm{pO}_{2}}$ ), and answers to the check-in questions. From this report, coaches can select a detailed daily report (see the supplementary materials) with histograms of the step count or metabolic equivalents, as well as detailed pulse oximetry data, for further surveillance. Additionally, coaches can select to see data from previous weeks.

There is a defined database schema for storing and retrieving de-identified participant activity, pulse oximetry, weather, and daily questionnaire data as well as supporting objects (eg, flags, alerts, messages). A representational state transfer web service was implemented to store and retrieve data.

\section{Pilot Study 1: System Evaluation by Users}

A pilot study of the devices and program was conducted before implementation of the prospective clinical trial. Pilot study 1 aimed to assess the ease of the system, particularly with regard to the technology, and to troubleshoot the system. This study has been approved by the Mayo Clinic institutional review board.

Three subjects with moderate-to-severe COPD took the system home for $5 \mathrm{~d}$. The participants were encouraged to exercise daily with the video and to view the help videos. The participants were contacted by telephone and were administered a questionnaire assessing their experience with the system and their adherence. Qualitative responses were transcribed verbatim.

\section{Outcome Measures}

Technology. A 7-item questionnaire assessed the ease of system use. Questions 1-5 assessed the participant's ability to use the tablet and access its various components in a yes/no format. The last 2 questions assessed the ease of using the technology and inquired whether the participant followed along with the exercise videos.

Adherence. We assessed whether participants completed the exercises, wore the pulse oximeter and activity monitor, and completed the check-in wellness questionnaire over the 5-d trial period. Adherence measures were assessed as a percentage.

Participant Opinions. Participants were asked to rate on a 10-point Likert-type scale how helpful they felt the study was and how easy the technology was to navigate. Higher scores reflect more positive evaluations. Participants were also asked to answer whether they would recommend the program to another patient and if they would continue using the system if it were available, on a yes/no scale.

Qualitative Feedback. Four questions assessed participant perceptions of the system, specifically their opinion on using the tablet, what they would change about the how-to videos, what they did not like about the system, and whether there is anything that should be done differently.

Participants. Subjects were recruited from the Mayo Clinic pulmonary clinic; patients having COPD and eligible for PR were invited to participate. Study participants were required to be $40 \mathrm{y}$ of age or older and have a diagnosis of Global Initiative for Chronic Obstructive Lung Disease (GOLD) stage II, III, or IV COPD documented by pulmonary function testing. Participants were required to be current or previous smokers, with at least 10 pack-years of cigarette smoking, and must have visited a Mayo facility at least once.

\section{Pilot Study 2: Feasibility of the Complete Program and Adherence}

Pilot study 2 aimed to assess the feasibility of the study (home PR plus health coaching) as well as participant adherence. After completing minor revisions, 12 participants were sent home with a complete system and were asked to complete the entirety of the study. This allowed the researchers to pilot the full program. This study has been approved by the Mayo Clinic institutional review board.

Participants. Pilot Study 2 had the same inclusion criteria as noted above in Pilot Study 1. 


\section{Home-Based Pulmonary Rehab and Health Coaching for COPD}

Study Timeline. This study was a single-group design with an intervention period lasting 8 weeks. During the trial period, subjects used the home-based PR system and had weekly PR health coaching calls.

Data Collection. Twelve participants consented and enrolled in the home PR program. Adherence data were obtained from the system. The Working Alliance InventoryShort Revised (WAI-SR) score was obtained from the questionnaire packet sent after completion of the intervention phase. After completion of the intervention, participants were contacted by telephone and administered a 5-question follow-up questionnaire.

\section{Outcome Measures}

Adherence. Daily completion data regarding the 12min walking, full-body exercises, and check-in wellness questionnaire were collected from the system for each participant. Adherence rates were calculated as a percentage of days the task was completed out of 48 possible days. The individual adherence rates were averaged to obtain the overall adherence rate for each intervention component: walking, exercises, and filling out the check-in questions. An overall study adherence rate was also computed.

WAI-SR. The WAI-SR is a 12 -item self-report measure of working alliance. ${ }^{18}$ It has 3 subscales: goals, tasks, and bond. Each WAI-SR subscale is scored on a 7-point Likert-type scale ranging from 1 (never) to 7 (always). Subscale scores can range from 4 to 28 and can, if desired, be summed to obtain a total score. Thus, total scores can range from 12 to 84 . Higher scores reflect more positive ratings of working alliance.

Five-Question Follow-Up Questionnaire. Items 1-4 were rated on a 7-point Likert-type scale and assessed the participants' perception of the home-based PR program. An answer of 7 reflected the most positive perception, and an answer of 1 represented the most negative perception. Item 5 was an open-ended, qualitative measure of the participants' likes and dislikes of the program.

\section{Results}

\section{Pilot Study 1: System Evaluation by Users}

Data means and SD values were calculated using Excel 2010 (Microsoft, Redmond, Washington). One hundred percent of participants reported that the technology of the system was easy to use. One hundred percent of participants were adherent with the expected use of the system: using the system technology, completing the exercises, and responding to the check-in well-being
Table 1. Quantitative Findings From Pilot Study 1 Regarding System Development

\begin{tabular}{lc}
\hline \multicolumn{1}{c}{ Parameters } & Responses \\
\hline Technology, \% & 100 \\
Used the tablet computer & 100 \\
Able to switch between different tablet screens & 100 \\
Able to view video on the tablet & 100 \\
Right amount of text on tablet & 100 \\
Watched how-to videos & 100 \\
Ease of using technology: easy & 100 \\
Used exercise video every time & \\
Adherence, \% & 100 \\
Completed well-being questionnaire on tablet & 100 \\
Completed exercises & 100 \\
Wore the pulse oximeter & 100 \\
$\quad$ Wore activity monitor & \\
Opinions & 8.67 \\
How helpful do you think this study will be?, & \\
$\quad$ 10-point scale & 9.67 \\
How easy was it to navigate the technology?, & 100 \\
$\quad$ 10-point scale & 66 \\
Recommend?, Yes \% & \\
Would you continue using it if available?, Yes \% &
\end{tabular}

questionnaire regarding energy, breathlessness, and overall well-being once daily for 5 consecutive days. On a 10-point scale, overall, the participants felt that the study was helpful (median $=9$, interquartile range $8-9$ ) and that the technology was easy to use (median $=9$, interquartile range 9-10). See Table 1 for a complete list of findings.

Overall, participants felt that the tablet was helpful and had diverse feedback regarding their experience with the system. See Table 2 for comprehensive participant feedback. The feedback helped to customize the final iteration of the system, with minor changes made. The system was able to both collect data locally and transmit it to the secure external cloud.

\section{Pilot Study 2: Feasibility of the Complete Program and Adherence}

Data means and SD values were calculated using Microsoft Excel 2010. The response rate was 100\%, resulting in a sample population of 12 participants.

Adherence. Participant $(n=12)$ adherence was $89 \pm 0.22 \%$ for the 12 -min walk, $88 \pm 0.20 \%$ for exercising, and $84 \pm 0.30 \%$ for completing the check-in wellness questionnaire for $6 \mathrm{~d} /$ week for 8 weeks. Overall study adherence was $87 \pm 0.24 \%$.

WAI-SR. One participant answered only one question on the WAI-SR and was thus omitted, yielding a sample 


\section{Home-Based Pulmonary Rehab and Health Coaching for COPD}

Table 2. Qualitative Findings From Pilot Study 1 Regarding System Development and Participant Likes and Dislikes

\begin{tabular}{|c|c|}
\hline Questions & Responses \\
\hline What did you think about using the tablet? & $\begin{array}{l}\text { "It helped to be able to follow along with someone, follow the pace." } \\
\text { "Very helpful. Very visual. [The] tablet was portable, which allows people who need visual } \\
\text { training to follow along." } \\
\text { "Wasn't that difficult, I could see where if someone would stick with it they would } \\
\text { benefit." }\end{array}$ \\
\hline How would you change the how-to videos? & $\begin{array}{l}\text { "When I would pause the [seated exercise] video sometimes it would take me back to the } \\
\text { beginning." } \\
\text { "The walking part should be able to skip the instructions, [but] keep the stand on each foot } \\
\text { part- - that is helpful. It helps with awareness and balance walking part. [You] should tell } \\
\text { the patients that each step is } 3 \text { seconds. I got dizzy trying to walk and follow the video, } \\
\text { until I discovered that each step was } 3 \text { seconds." } \\
\text { "I cannot remember the pursed lipped breathing video; I wish I could view that again. } \\
\text { Maybe have instructions in the how to section or make the video available with the } \\
\text { exercise videos." }\end{array}$ \\
\hline Were there things you didn't like? & $\begin{array}{l}\text { "The Garmin." } \\
\text { "The slow walking bothered me, but then when you explained to me that the slow walking } \\
\text { really helps me work on my balance, I really thought about it and plan to really work on } \\
\text { it." }\end{array}$ \\
\hline $\begin{array}{l}\text { Is there anything we could do differently or } \\
\text { that we need to change? }\end{array}$ & $\begin{array}{l}\text { "Can't think of anything. [My] main complaint is about the armbands and the Garmin." } \\
\text { "When demonstrating, do it on [the] patient's tablet so that they don't have to go home and } \\
\text { do [the] training again." } \\
\text { "I wish there could be a separate program for people who are able to tolerate more } \\
\text { exercise. It would be nice to have variation in the exercises. I don't think I could do the } \\
\text { same exercises for } 8 \text { weeks." }\end{array}$ \\
\hline
\end{tabular}

population of 11 participants. The group tested $79 \pm 3.43 \%$ of the task subscale (mean $=22.2), 89 \pm 2.21 \%$ of the bond subscale (mean $=24.9)$, and $79 \pm 4.38 \%$ of the goal subscale (mean $=21.8$ ). The sample population tested $82 \pm 6.59 \%$ on the overall working alliance inventory (mean $=68.9$ ).

Perceptions. The first question, "How do you rate the home-based pulmonary rehab program?" had a mean \pm SD rating of $6.2 \pm 0.94$ on a 7-point Likert-type scale $(n=12)$, with 1 representing the most negative response and 7 indicating the most positive response. The ease of operation had a mean \pm SD rating of $6.6 \pm 0.90(n=12)$. When asked about the impact the program had on the participants' personal life, the mean \pm SD response was $4.7 \pm 2.02(n=12)$. The participants mean \pm SD response to the question, "If this program were available now, how likely would you be in recommending this program to another patient with COPD?" was $6.4 \pm 1.00(n=12)$.

When asked about what the participants liked the most and the least about the program, the 3 common themes that emerged were: receiving support from the coach, the appropriateness of the exercises, and a lack of negative feedback. Common themes and quotations can be found in Table 3 . Although not frequent enough to be included as a common theme, 2 of the participants noted that the program gave them structure and helped regiment them, with one noting, "There were days that I didn't want to get up and do [the exercises]. . . but I did, because I made a commitment."

When asked what they liked least about the program, the sole common theme was that the participants had no negative feedback. Two participants did not like the slowwalking exercise, stating that "I just couldn't do it," and "I wasn't accustomed to [moving that slowly]." Another participant disliked the predetermined timing of the exercises, feeling that it was a "very slow pace." The fourth participant to provide negative feedback stated that, "Well, it took some of my time."

In the follow-up questionnaire, several (33.33\%) noted that they desired a disc with a copy of the exercises to follow along with after the intervention period ended and they no longer had access to the system. This desire was also expressed by one of the 3 participants in pilot study 1 . This request is currently being processed, and the materials will be available for those who enroll in the prospective clinical trial.

\section{Prospective Clinical Trial Design}

This prospective study will be a single-center, randomized, wait-list controlled trial that will recruit 120 participants and will be funded by the National Institutes of Health. The study will be conducted over a period of 24 months, following the timeline shown in Figure 2.

This study is primarily focused on the ability of remote PR to affect disease-specific quality of life, as measured 


\section{Home-Based Pulmonary Rehab and Health Coaching for COPD}

Table 3. Common Themes Regarding the Full Program From Pilot Study 2

\begin{tabular}{|c|c|c|}
\hline Common Themes & Response $(\%)$ & Participant Quotes \\
\hline Support from coach & $33 \%$ & $\begin{array}{l}\text { "[The health coach] keeping an eye on me." } \\
\text { "I feel like [the health coach] was very helpful to me, and she would answer any questions I } \\
\text { had. I would give her a ring and she guided me through running the program." } \\
\text { "Feeling that people care. I know it was a research thing, but it was nice to know that somebody } \\
\text { really cared." } \\
\text { "[Having] somebody checking in on me. That made me want to do it." }\end{array}$ \\
\hline Appropriateness of exercises & $75 \%$ & $\begin{array}{l}\text { "I was able to do it at my leisure. It was something I could manage from home." } \\
\text { "The slow walk. I thought that wasn't very important, but it really helps with your balance." } \\
\text { "I was doing harder exercises at the time, but you could see when you were done that they all } \\
\text { had a purpose." } \\
\text { "It encouraged me to relax more-the mindfulness part of it." } \\
\text { "It's a good way-it's a healthy way-of doing rehab, and it's not hard on you." } \\
\text { "It opened my eyes to what I should be doing. When I compare how I felt when I started with } \\
\text { how I felt when I ended, I was different-I had improved." } \\
\text { "It was neat keeping track of how many steps you take in a day, I had never done that. I thought } \\
\text { it was interesting and it regimented me." }\end{array}$ \\
\hline Lack of negative feedback & $58 \%$ & $\begin{array}{l}\text { "I really didn't dislike anything actually. I just found it very easy to do." } \\
\text { "There wasn't anything really that was a detriment or a deterrent. I just thought it was a pretty } \\
\text { good program." } \\
\text { "There was nothing I didn't like about the program-it was all good!" }\end{array}$ \\
\hline
\end{tabular}

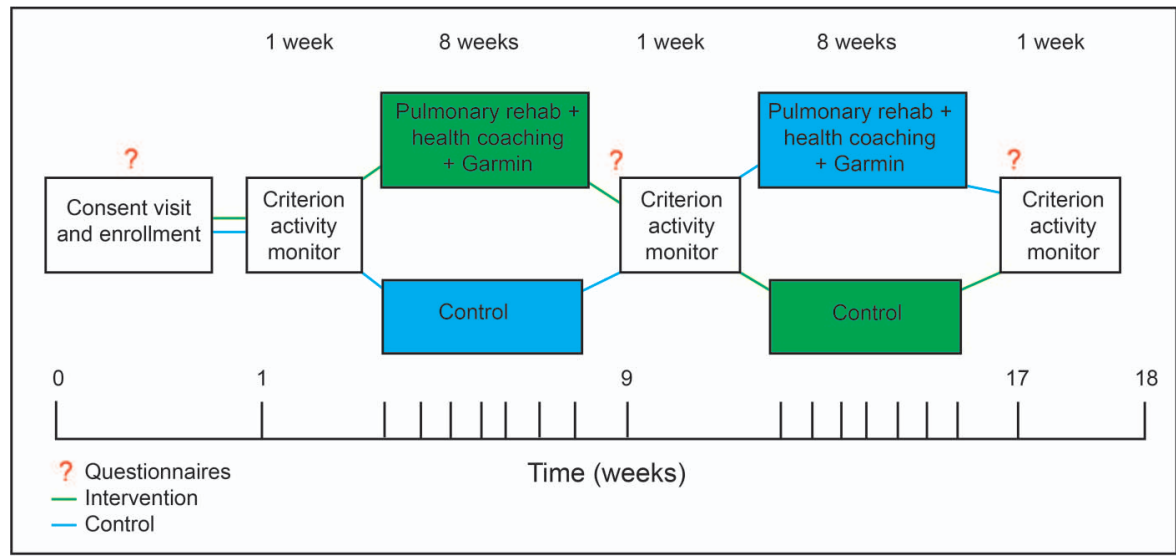

Fig. 2. Timeline for the prospective clinical trial.

by the Chronic Respiratory Disease Questionnaire with the aim of detecting a clinically meaningful difference of 0.5 points between groups. Secondary outcomes include selfmanagement support abilities, ratio of positive-to-negative emotions, and daily physical activity and sleep quality as measured by an accepted standard activity monitor. This activity monitor will not replace the Garmin activity monitor, which will be worn for the entirety of the intervention period. The study has been approved by the Mayo Clinic Rochester human subjects institutional review board.

\section{Discussion}

Results from the pilot studies indicate that the proposed program is feasible, that the home PR program was well- liked, and that adherence is very high. The latter may represent a seminal finding for PR research and eventually in clinical practice, as adherence is a critical and prevalent problem in PR. ${ }^{16} \mathrm{We}$ are striving for methods to create conditions for a behavior change, ${ }^{17}$ and this program may be doing something good, as the adherence to $6 \mathrm{~d} /$ week of practice was so high that it warrants not only further validation but also propose a novel concept in the field the synergy and possibly the effectiveness of an intervention that combines home-based PR with health coaching. Subjects expressed in both pilot studies that PR is about making a commitment to themselves and to the coach, a finding that is supported in our previous randomized study on health coaching. ${ }^{12}$ Subjects also expressed that proper technological and personal support strengthened their adherence as time passed. 


\section{Home-Based Pulmonary Rehab and Health Coaching for COPD}

The occurrence of the common theme "appropriateness of the exercises" in pilot study 2 indicates that the vast majority of the participants felt that the program was suited to their needs and interests. This reassuring feedback highlights the flexibility of the program: The exercises are simple, mild, and accommodating to all levels of exercise mastery. Whereas the entire PR program is beneficial, feedback and preliminary results from pilot study 2 highlight that there is an aspect of the program that will appeal to everyone. In addition, because daily physical activity has been recognized as a critical factor associated with survival in COPD, the home PR program created was geared more to promoting daily physical activity than to increasing exercise capacity. Thus, the aim of the program differs from typical PR programs often focused on exercise capacity in that it aims to improve physical activity rather than exercise capacity.

The proposed home PR program has been carefully crafted in our previous National Institute of Health awards. The incorporation of health coaching into the home PR program is rooted in our previous work in subjects with severe COPD, ${ }^{12}$ which has shown that this particular behavioral intervention is related to a decrease in hospitalizations. The preliminary results presented in this work support a seamless incorporation of this technology and behavior program into the daily care of COPD patients. With the widespread availability of relatively inexpensive tablet computers and monitoring devices, remote PR may be an effective way to increase patient participation and ongoing use of $\mathrm{PR}$ while still providing monitoring of both medical concerns and adherence.

A potential limitation in these pilot studies is the small sample size. However, as these studies were intended to gather information regarding participant feedback and adherence, a larger sample size is not as crucial as it is in a randomized clinical trial.

The proposed randomized study is funded by the National Institutes of Health and will be the first study to use home PR with comprehensive monitoring synergized with a behavior change intervention like health coaching to improve quality of life and physical activity in individuals with COPD. This paper documented the program development and preliminary results that suggest feasibility and acceptability. This paper also presented the comprehensive methods to be used in the upcoming randomized clinical trial. The results of the study will help to optimize and standardize self-management support programs in the context of home PR. The results will also facilitate dialogue about novel methods of PR and behavior change in their respective fields. Additionally, the use of commercially available devices for the proposed home PR program ensures its feasibility for widespread use.

One strength of the proposed randomized clinical trial is that this is the first multimodal intervention to incorporate a behavior change intervention (health coaching) and commercially available technology into a physician-developed home PR program with the aim of increasing quality of life and physical activity, both critical outcomes in COPD. ${ }^{19}$ Another strength is that quality of life and physical activity will be measured using validated tools and accelerometers. Preliminary evidence from the pilot studies reported on in this work indicates that adherence to PR, which continues to be a significant problem in COPD clinical practice, is something that a home PR program with a behavioral component may provide a solution for and thus make a significant impact in the field of COPD clinical practice.

A potential limitation of the prospective clinical trial is that the disentanglement of the effects of the home PR and of the health coaching will be impossible. However, given the lack of research regarding home PR and health coaching, to be able to determine the effectiveness of the combination is a significant movement forward. Additionally, this inability to disentangle these effects represents an opportunity for future research.

\section{Conclusions}

Program development and preliminary results from the 2 pilot studies presented in this work indicate that the proposed home PR program is both feasible and accepted by patients, thus promoting adherence. It is hoped that the prospective clinical trial will increase our understanding of how a simple, monitored home PR program plus a behavioral program (health coaching) can impact quality of life in individuals with COPD and how this improvement can be translated into increased physical activity levels. This trial will also prepare the field for comprehensive COPD patient management, particularly the synergy of behavioral intervention programs, like health coaching, with more established exercise-based programs, like PR. This trial has the potential to promote changes in improving homebased COPD management and to significantly impact patient overall well-being.

\section{REFERENCES}

1. Spruit MA, Singh SJ, Garvey C, ZuWallack R, Nici L, Rochester C, et al. An official American Thoracic Society/European Respiratory Society statement: key concepts and advances in pulmonary rehabilitation. Am J Respir Crit Care Med 2013;188(8):e13-e64.

2. Keating A, Lee A, Holland AE. What prevents people with chronic obstructive pulmonary disease from attending pulmonary rehabilitation? A systematic review. Chron Respir Dis 2011;8(2):89-99.

3. Holland A. Telehealth reduces hospital admission rates in patients with COPD. J Physiother 2013;59(2):129.

4. Holland AE, Hill CJ, Rochford P, Fiore J, Berlowitz DJ, McDonald CF. Telerehabilitation for people with chronic obstructive pulmonary disease: feasibility of a simple, real time model of supervised exercise training. J Telemed Telecare 2013;19(4):222-226.

5. Griffiths TL, Burr ML, Campbell IA, Lewis-Jenkins V, Mullins J, Shiels K, et al. Results at 1 year of outpatient multidisciplinary 


\section{Home-Based Pulmonary Rehab and Health Coaching for COPD}

pulmonary rehabilitation: a randomised controlled trial. Lancet 2000; 355(9201):362-368

6. Holland AE, Mahal A, Hill CJ, Lee AL, Burge AT, Moore R, et al. Benefits and costs of home-based pulmonary rehabilitation in chronic obstructive pulmonary disease: a multi-centre randomised controlled equivalence trial. BMC Pulm Med 2013(Can’t get issue from record);13:57.

7. Benzo R. Collaborative self-management in chronic obstructive pulmonary disease: learning ways to promote patient motivation and behavioral change. Chron Respir Dis 2012;9(4):257-258.

8. Bucknall CE, Miller G, Lloyd SM, Cleland J, McCluskey S, Cotton $\mathrm{M}$, et al. Glasgow supported self-management trial (GSuST) for patients with moderate to severe COPD: randomised controlled trial. BMJ 2012;344:e1060.

9. Greening NJ, Williams JE, Hussain SF, Harvey-Dunstan TC, Bankart MJ, Chaplin EJ, et al. An early rehabilitation intervention to enhance recovery during hospital admission for an exacerbation of chronic respiratory disease: randomised controlled trial. BMJ 2014; 349:g4315.

10. Benzo R, Vickers K, Ernst D, Tucker S, McEvoy C, Lorig K. Development and feasibility of a self-management intervention for chronic obstructive pulmonary disease delivered with motivational interviewing strategies. J Cardiopulm Rehabil Prev 2013;33(2):113123.

11. Benzo RP. Mindfulness and motivational interviewing: two candidate methods for promoting self-management. Chron Respir Dis 2013;10(3):175-182.
12. Benzo R, Vickers K, Novotny PJ, Tucker S, Hoult J, Neuenfeldt P, et al. Health coaching and chronic obstructive pulmonary disease re-hospitalization: a randomized study. Am J Respir Crit Care Med 2016;194(6):672-680.

13. Killingsworth MA, Gilbert DT. A wandering mind is an unhappy mind. Science 2010;330(6006):932-932.

14. Depew ZS, Novotny PJ, Benzo RP. How many steps are enough to avoid severe physical inactivity in patients with chronic obstructive pulmonary disease? Respirology 2012;17(6):1026-1027.

15. Demeyer H, Burtin C, Hornikx M, Camillo CA, Van Remoortel H, Langer D, et al. The minimal important difference in physical activity in patients with COPD. PLoS One 2016;11(4):e0154587.

16. Greening NJ. Non-adherence to peri-exacerbation pulmonary rehabilitation: the people have spoken. Chron Respir Dis 2015;12(1):3-4.

17. Effing TW, Vercoulen JH, Bourbeau J, Trappenburg J, Lenferink A, Cafarella P, et al. Definition of a COPD self-management intervention: International Expert Group consensus. Eur Respir J 2016;48(1): 46-54.

18. Munder T, Wilmers F, Leonhart R, Linster HW, Barth J. Working Alliance Inventory-Short Revised (WAI-SR): psychometric properties in outpatients and inpatients. Clin Psychol Psychother 2010; 17(3):231-239

19. Vestbo J, Hurd SS, Agustí AG, Jones PW, Vogelmeier C, Anzueto A, et al. Global strategy for the diagnosis, management, and prevention of chronic obstructive pulmonary disease: GOLD executive summary. Am J Respir Crit Care Med 2013;187(4):347-365.

This article is approved for Continuing Respiratory Care Education credit. For information and to obtain your CRCE

(free to AARC members) visit

www.rcjournal.com

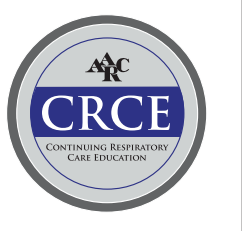

